\title{
Social Multimedia Computing
}

\author{
Peng Cui ${ }^{1}$, Lexing $\mathrm{Xie}^{2}$, Jitao Sang ${ }^{3}$, Changsheng $\mathrm{Xu}^{3}$ \\ 1 Tsinghua University \\ ${ }^{2}$ Australian National University \\ ${ }^{3}$ Institute of Automation, Chinese Academy of Sciences \\ cuip@tsinghua.edu.cn, lexing.xie@anu.edu.au, \{jtsang, csxu\}@nlpr.ia.ac.cn
}

\begin{abstract}
This article summarizes the corresponding full-day tutorial at ACM Multimedia 2014. This tutorial reviews recent progresses in social multimedia computing from two perspectives: social-sensed multimedia computing (3 hours) and user-centric social multimedia computing (3 hours).
\end{abstract}

\section{Categories and Subject Descriptors}

H.4.m [Information Systems Applications]: Miscellaneous

\section{General Terms}

Algorithms, Experimentation, Performance

\section{Keywords}

Social Media; Social Multimedia Computing

\section{INTRODUCTION}

Social media has revolutionized the way people share and access information, from work, daily life, to entertainment. With the ubiquitous presence of capturing devices, multimedia has become the major sharing and interacting medium for information access and communication in various social media networks. There is a clear trend in social media information services for adopting multimedia content. For example, Twitter increases its support on pictures and videos, by releasing its 6 -second video sharing app, Vine. Tencent's audio chatting tool, WeChat, has attracted more than 300 million users in less than two years, which is said to replace traditional SMS.

On the other hand, the prominence of social media has also witnessed the social trend in multimedia data generation and consumption. For example, Facebook reportedly sees 350 millions photos uploaded each day as of November 2013; 100 hours of video are uploaded to YouTube every minute, resulting in a total of more than 2 billion videos by

Permission to make digital or hard copies of part or all of this work for personal or classroom use is granted without fee provided that copies are not made or distributed for profit or commercial advantage, and that copies bear this notice and the full citation on the first page. Copyrights for third-party components of this work must be honored. For all other uses, contact the owner/author(s). Copyright is held by the author/owner(s).

MM'14, November 3-7, 2014, Orlando, Florida, USA.

ACM 978-1-4503-3063-3/14/11.

http://dx.doi.org/10.1145/2647868.2654852. the end of 2013. According to Alexa statistics, three out of the four fastest-growing social media websites around 20122013 focus on multimedia sharing. Moreover, social media gives birth to many new types of multimedia, e.g., image tweet, audio picture, geo-tagged video, etc. This significantly extends the scope and application areas of multimedia.

The multimedia data generated and consumed under social media circumstances is referred to as social multimedia. Three elements are identified, i.e., multimedia content, user, and interactions (between both user-user and user-content). Impacted by the participatory nature of WEB 2.0, users actively participate in the generation as well as consumption processes. As the hybrid of multimedia and social media, social multimedia enjoys advantages of both direct rich sensory simulation and efficient information access and propagation, thus having great potentials in analysis and utilization.

The emergence of social multimedia has brought challenges as well as opportunities to computing. On one hand, most social multimedia services are user-oriented, making it important to understand user demands from their interactions with the multimedia content. On the other hand, while multimedia content analysis still remains open, the participatory property of social multimedia offers a new solution perspective. Social multimedia computing, a multidisciplinary research and application field, has been developed to understand social multimedia content and connect the social multimedia content with users by exploiting the various social interactions. The potential applications range from information service, communication, entertainment, to healthcare, security, etc.

Thanks to the wide prevalence of social multimedia data and the increasing demands for social multimedia services, there has been a growing number of research on social multimedia computing, evidenced by the volume of papers produced, and many related tracks and special issues in prestigious multimedia conferences and journals. In this tutorial, we review the recent progresses in social multimedia computing from two perspectives.

\section{PART I: SOCIAL-SENSED MULTIMEDI- A COMPUTING}

The ultimate goal of multimedia computing is to deliver multimedia content to users according to their information needs (intentions). Multimedia computing can be decomposed into various stages: multimedia compression (for storage), multimedia communication (for delivery), and multimedia content analysis (for intelligence). Among these, multimedia analysis has become mainstream in the multi- 
media community, and related technologies have advanced significantly since the end of the last century. However, in addition to the notorious Semantic Gap, how to bridge the multimedia content with end users, the lastmile technology for multimedia services, is rarely researched. This lack of understanding directly causes an obvious Intention Gap between multimedia data and the real information needs of users, which has become a bottle-neck in advancing intelligent multimedia computing technologies for use in real applications.

Users' (both crowds' and individuals') intention-related information (including long-term interests, instantaneous intentions, and emotions, etc), their behavior patterns, and ultimately, the common principles of user-multimedia interactions under different contexts can all be sensed from social media, which are summarized by social knowledge on usermultimedia interactions. It is these social knowledge that reflects user needs and establishes a bridge for multimedia data and user needs. How to organically integrate multimedia data, user needs and social knowledge into multimedia computing technology is a critical issue. Here, we propose a new multimedia computing paradigm, social-sensed multimedia computing, to integrate all the recent works that bring social media, a valuable source of sensing user needs and social knowledge, into the loop of multimedia computing. It opens a brand new arena for the multimedia research community with a potentially big impact in both academia and industry.

\section{PART II: USER-CENTRIC SOCIAL MUL- TIMEDIA COMPUTING}

Social multimedia computing is very different from traditional and web multimedia computing. Web multimedia computing is heavily related to the WEB1.0 environment, which is dominated by broadcast media developed by professional designers for passive users. In traditional multimedia computing, the analysis focus is the multimedia content, and the goal is content understanding and application, e.g., media content analysis, semantic classification and annotation, structured media authoring. On the contrary, social multimedia computing has an obvious user-centric characteristic: (1) User is the basic data collection unit. Viewing each user as a data sensor, social multimedia is constituted by what users see, listen, think, feel and speak. Moreover, user bridges multimedia network and social network, contributing to most of the social interactions in social multimedia. (2) User is the ultimate information service target. As discussed above, social multimedia services are user-oriented and have a customized trend. Understanding the personalized demands is critical to most social multimedia computing problems.

We present the user-centric research paradigm for social multimedia computing, and organize the related work into three basic tasks of "From users, On users, and For users": (1) From users: user-perceptive multimedia content analysis. Users' social interactions capture what they perceive the multimedia content and can be exploited towards multimedia content analysis. For example, user-contributed picture tags indicate user-perceived visual semantics, user browsing behaviors, such as pause, fast-forward, indicate video structure information. (2) On users: social multimedia activitybased user modeling. Online activities reveals important clues of user background information and are utilized for user modeling of demographic facts, personal interests, social network status, mobility patterns, consuming patters, emotional orientation, etc. (3) For users: customized multimedia services. Given results from user-perceptive multimedia content analysis and user modeling, customized multimedia services are developed to satisfy personalized needs.

\section{TUTORIAL OUTLINE}

The tutorial will start with a brief joint background section. The following introduces the detailed topics addressed in the two parts, respectively.

\section{Part I: Social-sensed Multimedia Computing}

1. Introduction

a). Current status of multimedia computing

b). The paradigm of social-sensed multimedia computing

c). Basic problems and key challenges

2. Sensing user models and behavior patterns from social media

a). User profiling from social media

b). User behavioral analysis and modeling

c). Social graph mining

3. Integrating social knowledge with multimedia computing

a). Social factor assisted multimedia content understanding

b). Social-sensed multimedia search and recommendation

c). Multimedia affective computing

4. Discussions and future directions

Part II: User-centric Social Multimedia Comput-
ing

1. Introduction to user-centric social multimedia computing

2. User-perceptive multimedia content analysis (MCA)

a). MCA based on user-generated metadata

b). MCA based on user-multimedia interaction

c). MCA based on user-user interaction

3. Social multimedia activity-based user modeling

a). User interest modeling

b). User demographics inference

c). User social relation analysis

4. Customized multimedia services

a). Customized multimedia services: challenges

b). Customized multimedia services: applications

5. User-centric cross-network multimedia computing

a). Cross-network knowledge association mining

b). Cross-network user behavior analysis

c). Cross-network collaborated applications

d). Discussions and future directions 